\title{
Synthesis and Characterization of Poly-o-Toluidine: Kinetic and Structural Aspects
}

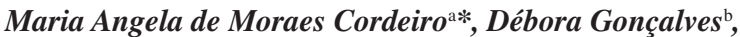 \\ Luis Otavio de Sousa Bulhões ${ }^{\mathrm{c}}$,João Manuel Marques Cordeiro ${ }^{\mathrm{a}}$ \\ aDepartamento de Física e Química, Faculdade de Engenharia de Ilha Solteira, \\ Universidade Estadual Paulista "Julio de Mesquita Filho", \\ Av. Brasil, 56, 15385-000 Ilha Solteira - SP, Brazil \\ 'Instituto de Física de São Carlos, Universidade de São Paulo, São Carlos - SP, Brazil \\ ${ }^{c}$ Departamento de Química, Universidade Federal de São Carlos, São Carlos - SP, Brazil
}

Received: March 13, 2003; Revised: November 20, 2004

\begin{abstract}
Poly $(o$-methylaniline) (poly-o-toluidine, PTOL) was synthesized by chemical oxidation of $o$-toluidine with ammonium peroxydisulfate in an aqueous $1.0 \mathrm{~mol} \mathrm{~L}^{-1} \mathrm{HCl}$ solution. The progress of polymerization was followed by measuring the open-circuit potential (OCP) of a Pt electrode immersed in the reaction medium with the polymerization time. The chemical synthesis of PTOL was carried out at different monomer:oxidant (M:O) molar ratios $(4: 1,2: 1,1.5: 1,1: 1$, and $0.66: 1)$, and the products obtained were characterized by infrared spectroscopy, gel permeation chromatography, and X-ray diffraction. The molecular weight and percentage of crystallinity of PTOL are higher for samples synthesized in an excess of the monomer, i.e. at higher M:O ratios. However, the yield of PTOL prepared at higher M:O ratios is considerably low, in particular at a 4:1 M:O ratio, which is the $\mathrm{M}: \mathrm{O}$ ratio most commonly used in the literature to synthesize polyaniline and its derivatives.
\end{abstract}

\section{Keywords: poly-o-toluidine, polymerization mechanism, open-circuit potential, polyaniline derivatives}

\section{Introduction}

A number of conducting polymers has been extensively studied in the last decades, in particular because of the great possibilities of application they offer such as electronic and optical devices, and batteries ${ }^{1,2}$. Polyaniline (PANI) is by far the most investigated conducting polymer, and it can be usually synthesized by chemical or electrochemical oxidation of aniline in aqueous and non-aqueous media ${ }^{3}$. The main difference between these two methodologies of synthesis is that the electropolymerization of aniline allows one to obtain details on the processes of charge transfer of a film directly deposited on an electrode surface, whereas the chemical polymerization of aniline in the presence of a strong oxidizing agent is suitable when a large quantity of a powdery material is required. The disadvantage of the chemical oxidation methodology is that chemically synthesized PANI exhibits a limited solubility in most common organic solvents, making it more difficult the preparation of cast films to be carried out. As a result, substituted PANIs ${ }^{4-7}$ have been synthesized in order to improve solubility of this class of polymers; yet, as a general rule, most of these derivatives exhibit characteristically low values of molecular weight and electric conductivity.

Many papers have been published on the progress of the electrochemical and chemical polymerization of aniline and derivatives in order to simultaneously correlate mechanisms of oxidation of aniline and properties of PANI such as electrical conductivity, molecular weight, and crystallinity ${ }^{4-10}$. However, these works have been commonly concerned with the kinetics of electrochemical polymerization of aniline, in which the rate-limiting step has been attributed to the formation of radical cations ${ }^{8-10}$. The following steps of this mechanism have not been well established so far, and different proposals have been suggested for the final stages of the polymer growth ${ }^{11-12}$. Regarding the chemical polymerization of aniline, it has been studied by varying the oxidant agent $t^{13}$, acid medium ${ }^{14-16}$, reaction temperature ${ }^{16,17}$, monomer:oxidant $(\mathrm{M}: \mathrm{O})$ molar ratios ${ }^{18-19}$, and reaction time ${ }^{20}$. But in spite of all these inquiries, the mechanism and kinetics of chemical polymerization of aniline and its derivatives have been not clearly understood yet, even if a great number of techniques have been used to follow the stages of polymerization of aniline at different synthesis protocols. One of these techniques is the measurement and analysis of open-circuit potential $(\mathrm{OCP})^{19-23}$ transients of an inert electrode (usually $\mathrm{Pt}$ ) put into to the reaction medium.

Our contribution in this paper was to study how the chemical synthesis of a soluble PANI derivative, poly-o-toluidine, PTOL, takes place at five different monomer:oxidant (M:O) ratios, focusing on results of open-circuit potential (OCP) transients, reaction yield, infrared spectroscopy, molecular weight, and crystallinity. Although different authors have already reported results on the electrical and optical properties of PTOL ${ }^{24-26}$, in particular as blends and fibers, no one has yet discuss how synthesis, kinetics of polymerization, and properties of PTOL can be correlated to each other. Aiming to fulfill this gap, we discussed the mechanism of chemical polymerization of $o$-toluidine in order to understand general aspects of the chemical polymerization processes of aniline and its derivatives.

\section{Experimental}

$o$-Toluidine was distilled under vacuum prior to be used. Reagent grade analytical ammonium peroxydisulfate, $\left(\mathrm{NH}_{4}\right)_{2} \mathrm{~S}_{2} \mathrm{O}_{8}$, and $\mathrm{HCl}$ were used as received. $o$-Toluidine (monomer, $\mathrm{M}$ ) and $\left(\mathrm{NH}_{4}\right)_{2} \mathrm{~S}_{2} \mathrm{O}_{8}$ (oxidant, $\mathrm{O}$ ) solutions were prepared at $0.25 \mathrm{~mol} \mathrm{~L}^{-1}$ in an aqueous $1.0 \mathrm{~mol} \mathrm{~L}^{-1} \mathrm{HCl}$ solution. Appropriated volumes of these solutions were taken in order to prepare mixtures of $4: 1,2: 1,1.5: 1,1: 1$, and 0.66:1 M:O ratios up to reach a total volume of $250 \mathrm{~mL}$ for each synthesis. All solutions and reaction media were kept at $0{ }^{\circ} \mathrm{C}$ by using a thermostatic bath. For a typical synthesis, the oxidant solution was added to the aniline solution by using a peristaltic pump at a constant rate of $20 \mu \mathrm{L} \mathrm{s}^{-1}$, with keeping constant the stirring rate for 
all M:O ratios. The total time for each synthesis was 5 hours, except for two syntheses, which were carried out for 18 hours for the sake of comparison. The ending point of each synthesis was chosen taking into account the shape of the OCP $v s$. reaction time curves.

During the polymerization reaction progress, the OCP was measured using a Pt plate $\left(\mathrm{ca} 1 \mathrm{~cm}^{2}\right)$ as the working electrode, and a hydrogen electrode in the same solution (RHE) as the reference electrode. Upon finished all syntheses, the precipitates obtained were filtrated under vacuum using a filter paper with small pores. After this step, the filtrated materials were dispersed in a $0.1 \mathrm{~mol} \mathrm{~L}^{-1} \mathrm{HCl}$ solution and then, re-filtrated. This procedure was repeated up to obtain a colorless filtrate, which means that oligomers of low molecular weights were removed as soluble fractions. Doped PTOLs were dried under dynamic vacuum at room temperature for 48 hours, after that, the reaction yields were calculated.

For molecular weight determination, dried, doped PTOL was deprotonated by stirring in a $0.1 \mathrm{~mol} \mathrm{~L}^{-1} \mathrm{NH}_{4} \mathrm{OH}$ solution for 24 hours, filtrated, and dried under dynamic vacuum at room temperature for 24 hours. A gel permeation chromatographer (GPC) from Waters Associates 440 with UV-VIS and refraction detectors was used. The molecular weight was measured by using an ultra styragel column $(300 \times 7.8 \mathrm{~mm})$ at $30^{\circ} \mathrm{C}$ and a flow rate of tetrahydrofuran (THF) as the carrier solvent of $1 \mathrm{~mL}$ minutes ${ }^{-1}$. The $\mathrm{UV}$ detector was set to $325 \mathrm{~nm}$ in order to monitor the elution of PTOL. PTOL solutions in THF at $1 \% \mathrm{wt}$ were prepared by stirring for 12 hours, followed by a filtration through a $0.5 \mu \mathrm{m}$ filter prior to injection into the column. The injection coil volume was $100 \mu \mathrm{L}$. Polystyrene Waters molecular weight standards ( 580 to $1,070,000 \mathrm{~g} \mathrm{~mol}^{-1}$ ) were obtained at $1 \% \mathrm{wt}$ in a degassed HPLC grade THF. The correlation coefficient for the calibration curve was superior to 0.999 to polystyrene. The distribution curve of molecular weights was obtained with Millenium 2010 software.

The FT-IR spectra were taken in a Bomem MB102C3 spectrophotometer with a resolution of 4.00 to $0.125 \mathrm{~cm}^{-1}$. Dried PTOL $(0.3 \mathrm{mg})$ was mixed with $\mathrm{KBr}(200 \mathrm{mg})$ and pressed under vacuum, and air was used as reference.

The WAXD scans were obtained using a Siemens D5000 X-ray diffraction instrument (K $\alpha 1.54056 \AA)$. Pure PTOL pellets were scanned at $0.4^{\circ}$ minutes $^{-1}$ for $2 \theta$ from 5 to $40^{\circ}$. The baseline was carried out using silicon oxide, and the percentage of crystallinity was calculated from the ratio between the values of the crystalline areas and the total area, which were taken from the diffractograms of PTOL after convolution.

\section{Results and Discussion}

Two typical OCP transients for a Pt electrode, which were measured during the progress of chemical polymerization of $o$-toluidine at 4:1 and 0.66:1 M:O ratios, are presented in Figure 1a. In these curves, it can be observed that the potential increases during the first minutes of the reaction (A-B), reaches a constant value (plateau) (BC), and then decays to a constant value as the reaction is completed (D). Similar features were obtained for the OCP curves at the M:O ratios of 2:1, 1.5:1 and 1:1 (results not shown). Figure 1b shows how the reaction time for the plateau region ( $\mathrm{tBC}$ ) varies as a function of the $\mathrm{M}: \mathrm{O}$ ratio.

Each part of the OCP curves in Figure 1a can be related to different stages of the $o$-toluidine polymerization, in a similar manner to that previously described for aniline ${ }^{27}$. Point $\mathbf{A}$ in the OCP curves corresponds to the potential of a Pt electrode at $c a 0.69 \mathrm{~V} v s$. RHE in an aqueous $\mathrm{HCl}$ solution containing $o$-toluidine, before addition of the oxidant solution. After initiating the addition of the oxidant solution, at a rate that was kept constant for all M:O ratios, the potential increased rapidly from $0.69 \mathrm{~V}$ to $c a 0.90-0.95 \mathrm{~V} v s$. RHE at point B. During these first seconds of the reaction, a pink color was observed in solution, indicating the presence of soluble oxidized oligomers. At the B-C plateau, precipitation of PTOL began, with the potential remaining at a constant value for a Pt electrode covered with a PTOL film in the pernigraniline form, which is one of the oxidation states of PANI and its derivatives. Pernigraniline, which was previously isolated and identified by elemental analysis at the B-C stage for aniline ${ }^{27}$, acts as an oxidant that is strong enough to oxidize monomers and oligomers in solution. At the same time, pernigraniline is reduced to the half-oxidized emeraldine form ${ }^{28}$. Finally, between points $\mathbf{C}$ and $\mathbf{D}$, a constant potential was reached at ca $0.69 \mathrm{~V} v s$. RHE for the Pt electrode covered with a PTOL film in the emeraldine salt form. At this stage, the polymerization reaction is completed and precipitation of PTOL takes place. In the literature, it has been shown that the oxidant concentration, which was continuously monitored during the recording of the OCP curves for aniline, is essentially depleted at point $\mathbf{D}^{27}$. Visually, it was noticed that the electrode surface becomes covered by a thin film of PTOL as soon as the polymerization reaction initiates, and this strongly bound film exhibits uniform texture, good quality and a green coloration at the end of polymerization. Furthermore, doped PTOL that was formed as a dark, solid precipitate at point $\mathbf{D}$ was washed, filtrated, and dried in order to calculate the reaction yield.

Compared to earlier OCP results for aniline ${ }^{21,27}$, the most visible difference noticed in the OCP curves for $o$-toluidine is the longer reaction time in the plateau region ( $\mathrm{tBC}$ ). Such longer values of $\mathrm{tBC}$ can be justified by considering that steric hindrance of $-\mathrm{CH}_{3}$ groups on the $o$-toluidine molecule occurs, which makes the occurrence of coupling reactions among species involved in the growth of the polymer chains more difficult. This also explains why the decay times of the OCP to a constant value (point $\mathbf{D}$ ) are considerably lower than the values obtained for aniline. In addition, this explains why low molecular weights are obtained for PTOL, as will be discussed later.

Considering of the dependence of $\mathrm{tBC}$ on the $\mathrm{M}: \mathrm{O}$ ratio, when there is an excess of the monomer in solution (relative to the oxidant), $\mathrm{t}_{\mathbf{B C}}$ decreases (see Figure 1b), indicating that the polymerization rate is faster at higher M:O ratios (e.g. at a 4:1 M:O). In this case, since consumption of the oxidant is faster in a solution containing an excess of the monomer, it can be assumed that $o$-toluidine remains in solution without reacting even after $c a 120$ minutes of reaction (point $\mathbf{D}$ in the OCP curves), and explains why a lesser amount of precipitate is obtained at a 4:1 M:O ratio.

After 5 hours of polymerization, the PTOL yield varies markedly with the M:O ratio, as can be better visualized in Figure 2a. Figure 2b shows the polymerization yield as a function of the relative amount of oxidant added to the reaction medium. As illustrated in the OCP curves, a total reaction time of $c a 5$ hours (300 minutes) is sufficient for the polymerization of $o$-toluidine to be competed (at point $\mathbf{D}$ in the OCP curves), independently of the $\mathrm{M}: \mathrm{O}$ ratio. For comparison, the depletion time was extended to 18 hours (1080 minutes) instead of 5 hours, but since similar yields were obtained (see additional points in Figure 2a), it can be inferred that the total reaction time has a little influence on the reaction yield.

Despite the lower yields obtained for PTOL at higher M:O ratios (e.g. $17 \%$ and $23 \%$ at 4:1 and 2:1 M:O ratios, respectively), PANI and its derivatives are usually chemically synthesized in the presence of an excess of the monomer ${ }^{13,21,29}$. The presence of a certain amount of residual monomer at the end of polymerization is usually justified by the higher electrical conductivity and molecular weights (MW) that are obtained in the presence of an excess of the monomer ${ }^{29,30}$. In fact, higher values of MW for PTOL synthesized at higher M:O ratios were also obtained, with the yield of PTOL decreasing in the opposite way (Figure 3). 


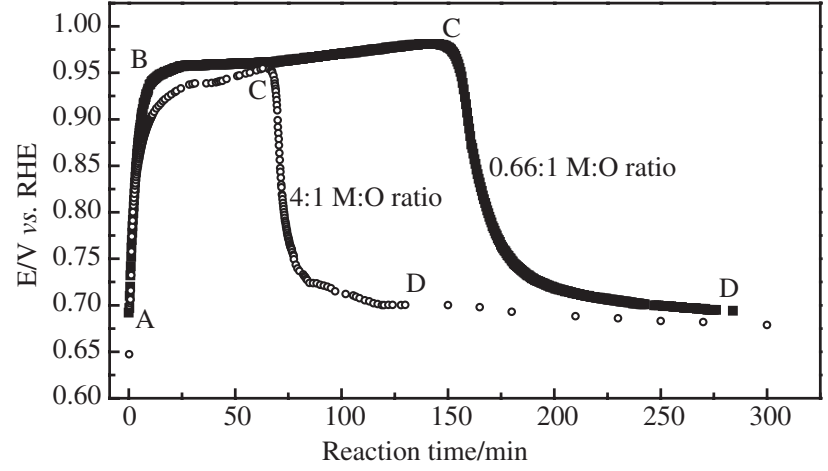

(a)

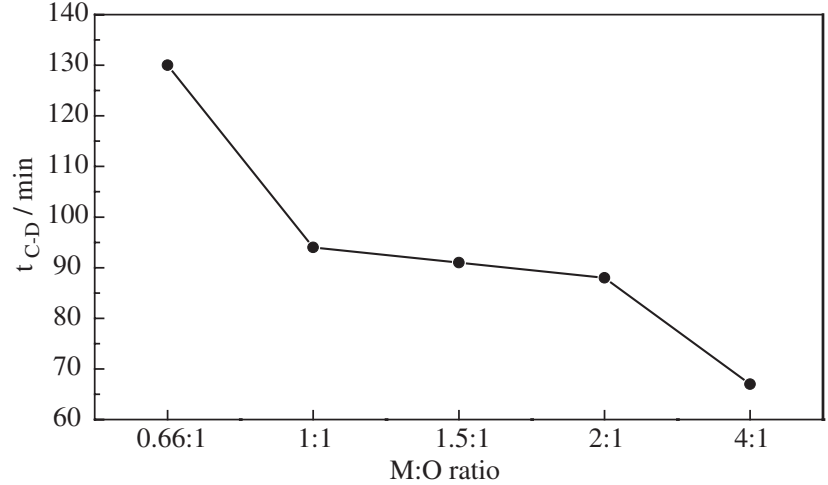

(b)

Figura 1. a) Curves of OCP vs. reaction time of Pt electrodes measured during the polymerization progress of $o$-toluidine at 4:1 and 0.66:1 M:O ratios; b) Values of time at the plateau region (tB-C) taken from the OCP curves at different M:O ratios.

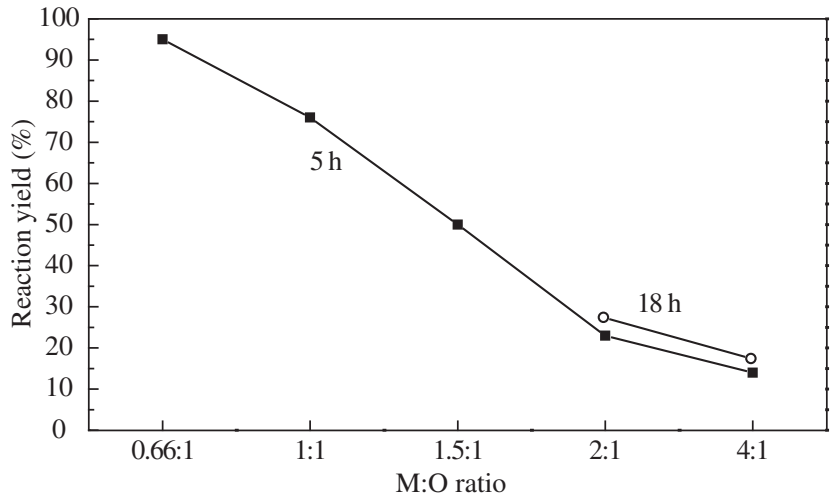

(a)

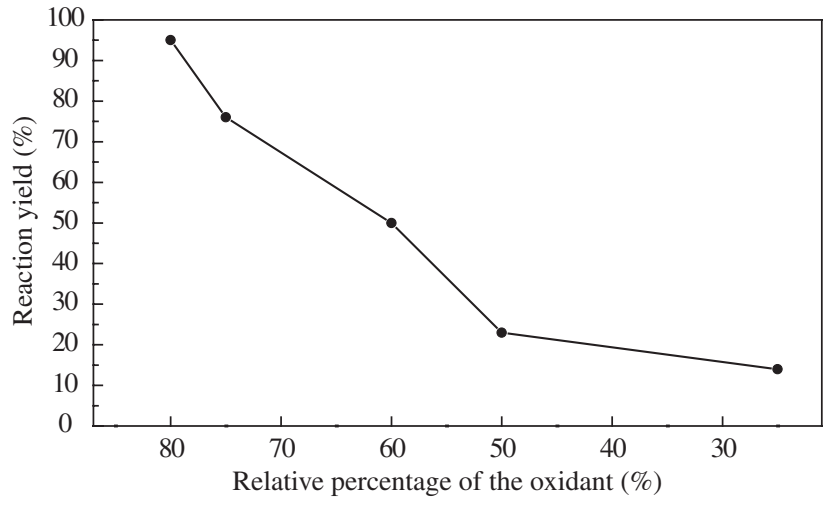

(b)

Figure 2. Yield of PTOL as a function of the, a) M:O ratio used in the synthesis; b) relative percentage of the oxidant added to the reaction medium.

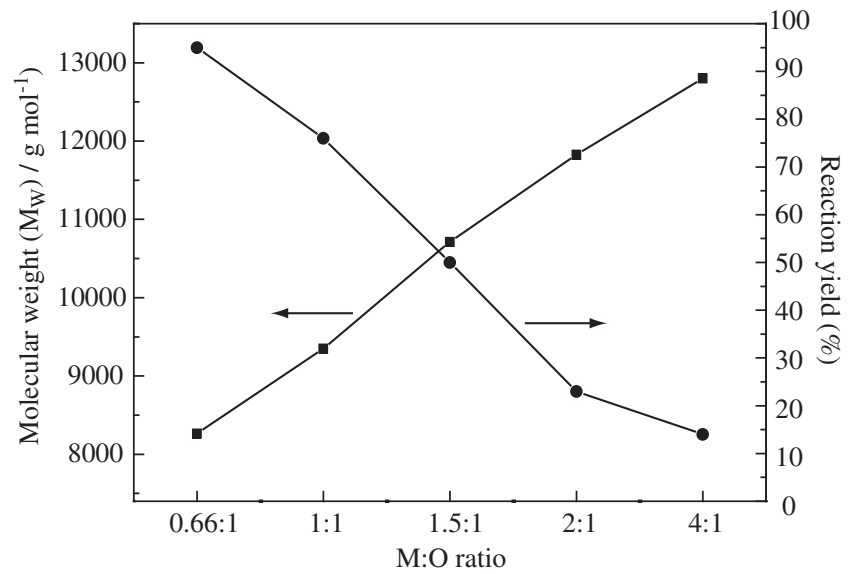

Figure 3. Values of molecular weight and reaction yield as function of the M: $\mathrm{O}$ ratio used in the synthesis of PTOL.

The values of MW seen in Figure 3 are characteristically low because they correspond to the low MW fraction of PTOL. These values were obtained by using tetrahydrofuran (THF), in which deprotonated PTOL is easily dissolved. Practically no amount of insoluble residue was observed in the PTOL solutions in THF, differing from PANI, which dissolves only partially in $\mathrm{THF}^{31}$. The gel permeation chromatograms of PTOL in THF showed a unimodal shape for all M:O ratios, with an MW fraction ranging from $\mathrm{ca} 8000$ to $13000 \mathrm{~g} \mathrm{~mol}^{-1}$ based on the polystyrene calibration. In the literature, there are a few studies of GPC analysis of substituted PANIs ${ }^{21,32}$. Mattoso et al. ${ }^{32}$ obtained a value of MW of $47600 \mathrm{~g} \mathrm{~mol}^{-1}$ for PTOL synthesized at a $4: 1 \mathrm{M}: \mathrm{O}$ ratio using a mixture of $0.5 \% \mathrm{LiCl} / \mathrm{NMP}$ as the mobile phase. However, studies in the literature have shown that effects of aggregation of PANI take place in diluted $\mathrm{LiCl} / \mathrm{NMP}$ solutions ${ }^{33}$, and it may lead to a colloidal rather than a molecular solution, artificially higher values of MW, and values of MW depending on the Li salt used as the additive (e.g. $\mathrm{LiCl}, \mathrm{LiBr}$, and $\left.\mathrm{LiBF}_{4}\right)^{34}$. Thus, it is possible to obtain different MW values for PANI using different solvents and preparation methods, with many parameters influencing such MW determinations. However, it can be inferred that independently of the carrier solvent chosen, both high and low MW fractions for PTOL follow the same tendency after varying the M:O ratio (i.e. increase from 0.66 to 4:1) as can be seen in Figure 3.

PTOLs prepared at different M:O ratios were structurally characterized by IR spectroscopy and X-ray diffraction. Figure 4 presents the IR spectrum and X-ray diffractogram of PTOL synthesized at a 4:1 M:O ratio. All spectra and diffractograms of PTOL produced at different $\mathrm{M}: \mathrm{O}$ ratios are similar and are not shown here. The IR results indicate that different $\mathrm{M}: \mathrm{O}$ ratios do not necessarily result in the production of different PTOL structures. As similarly described for PANI ${ }^{35,36}$, the main peaks seen in the spectra of PTOL can be assigned as follows: C-N stretch of the quinone structures at $1600 \mathrm{~cm}^{-1}$, $\mathrm{C}-\mathrm{N}$ stretch of benzene rings at $1498 \mathrm{~cm}^{-1}, \mathrm{C}-\mathrm{N}$ stretch of aromatic 
amines at $1380-1240 \mathrm{~cm}^{-1}$, and out of plane $\mathrm{C}-\mathrm{H}$ bending mode at $807 \mathrm{~cm}^{-1}$ (arrows in Figure 4a).

The X-ray spectrum of PTOL resembles that of $\mathrm{PANI}^{37}$, where a semi-crystalline profile for PTOL can be observed, independently of the M:O ratio used. The spectrum in Figure $4 \mathrm{~b}$ exhibits intense crystalline peaks for $2 \theta$ from $24^{\circ}$ to $28^{\circ}$ and weakly intense peaks between $8^{\circ}$ to $10^{\circ}$ and $12^{\circ}$ to $14^{\circ}$. In the literature $e^{38}$, the percentage of crystallinity of PANI, calculated by using the Manjunath equation ${ }^{39}$, has been shown to be dependent on the synthesis procedure used. It has been observed that doping with aqueous $\mathrm{HCl}$ helped PANI to become more crystalline, and this effect was also noticed in the present study with PTOL. The authors ${ }^{38}$ obtained a crystallinity of $45 \%$ and $74 \%$ for undoped and $\mathrm{HCl}$-doped PANI samples, respectively. It was observed that the higher the degree of crystallinity of PANI, the higher its electrical conductivity. Also, in the cited work, diffraction peaks for PANI appeared at $2 \theta=9^{\circ}, 15^{\circ}, 21^{\circ}$ and $25.6^{\circ}$, which are values very close to those obtained for PTOL here, although the $\mathrm{M}: \mathrm{O}$ ratio used in the chemical synthesis of PANI was not mentioned ${ }^{38}$.

In Figure 5, the results of crystallinity, molecular weight (MW) and polydispersivity $\left(\mathrm{M}_{w} / \mathrm{M}_{\mathrm{n}}\right)$ for PTOLs obtained at different M:O ratios after 5 hours and 18 hours of synthesis are compared. In this figure, it can be observed that there is a tendency for enhancement of the crystallinity with the MW, an effect already observed for other PANIs ${ }^{30,37}$. As a general feature, it can be asserted that the same type of correlation seems to be valid between MW and electrical conductiv- ity $^{30,38}$, as has been previously verified for PANI and its derivatives. It is interesting to observe the lower percentage of crystallinity obtained for PTOL that was kept in the reaction medium for a longer period of time $(18 \mathrm{~h}$ ) (see additional points in Figure 5a). Under such conditions, it can be assumed that the effects of chemical degradation of PTOL take place after a prolonged exposure to the reaction medium, despite the similar reaction yields that were obtained for syntheses carried out for $5 \mathrm{~h}$ and 18 hours (see Figure 2). In the latter case, a lower value of $\mathrm{MW}\left(9,157 \mathrm{~g} \mathrm{~mol}^{-1}\right)$, a lower percentage of crystallinity $(29.1 \%)$ and higher polydispersivity $\left(\mathrm{M}_{\mathrm{w}} / \mathrm{M}_{\mathrm{n}}=3.0\right)$ were obtained. This indicates that the longer reaction time is not recommended for the chemical synthesis of PTOL.

Based on the results presented here, the mechanism of chemical polymerization of $o$-toluidine can be summarized as follows. Basically, the OCP measurements indicate that the stages of polymerization of aniline and its derivatives (including $o$-toluidine) can be described by a sequence of reactions involving oxidation and coupling reactions at the $\mathrm{Pt} / \mathrm{solution}$ interface. The first step of this mechanism is the oxidation of $o$-toluidine to radical cations. As a subsequent step, the radical species couple with other radicals or neutral monomers to form dimers or radical dimers, respectively. After these steps, the neutral dimers are oxidized by the oxidant, which was added to the reaction medium. This results in radicals that couple either with radicals or other neutral molecules to form neutral oligomers or larger radicals, respectively. These oligomers are then reoxidized, and this implies

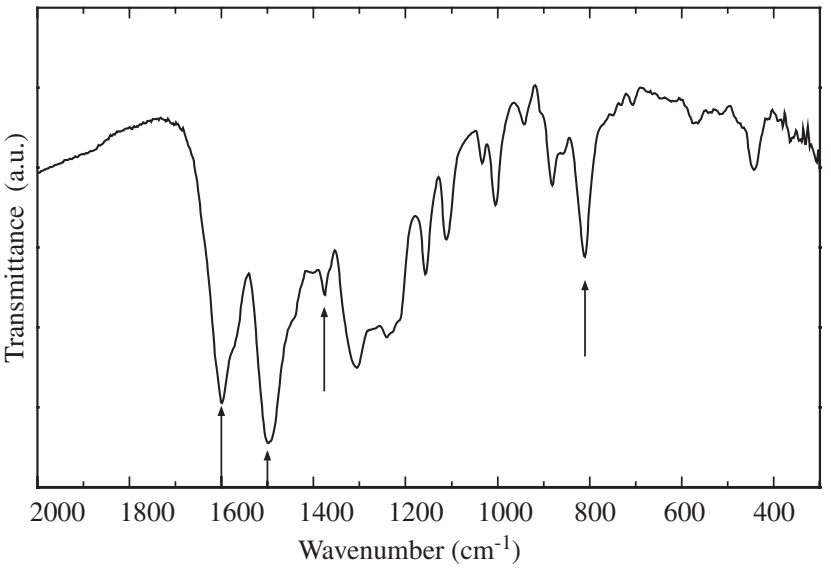

(a)

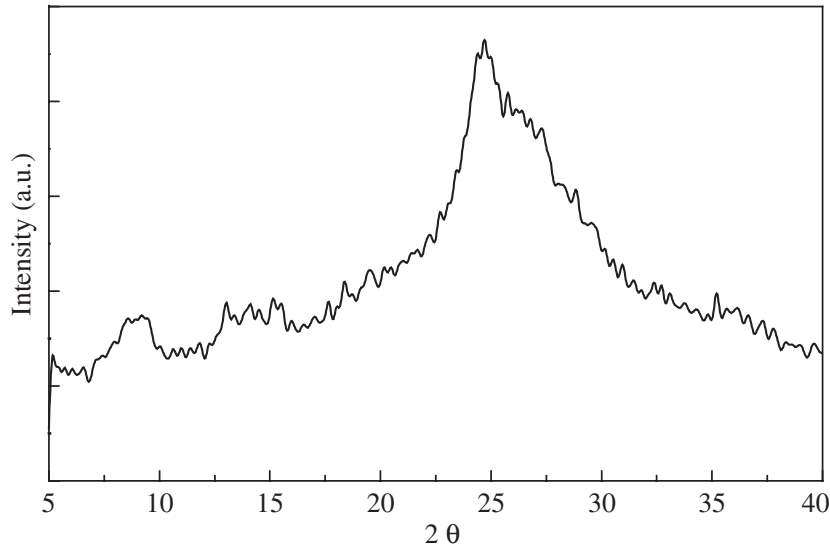

(b)

Figure 4. a) Infrared spectra of powdery, deprotonated PTOL obtained at a 4:1 M:O molar ratio; b) wide-angle X-ray diffractogram of powdery PTOL doped with a $\mathrm{HCl} 1.0 \mathrm{~mol} \mathrm{~L}^{-1}$ solution.

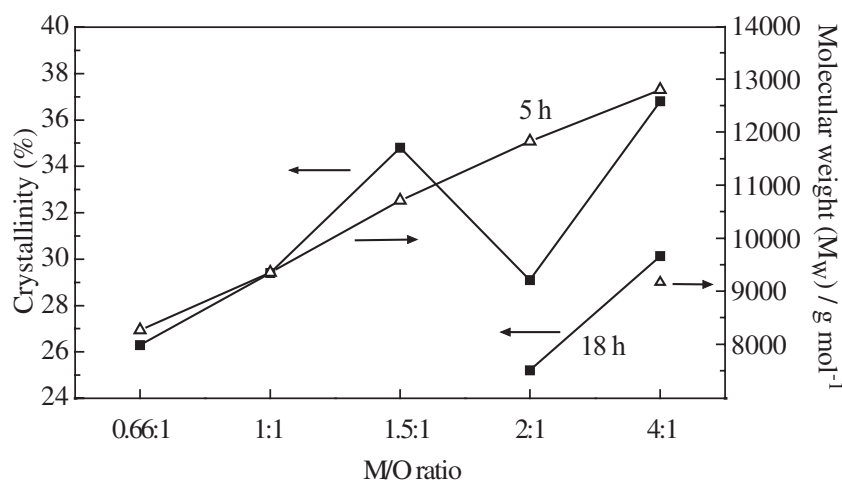

(a)

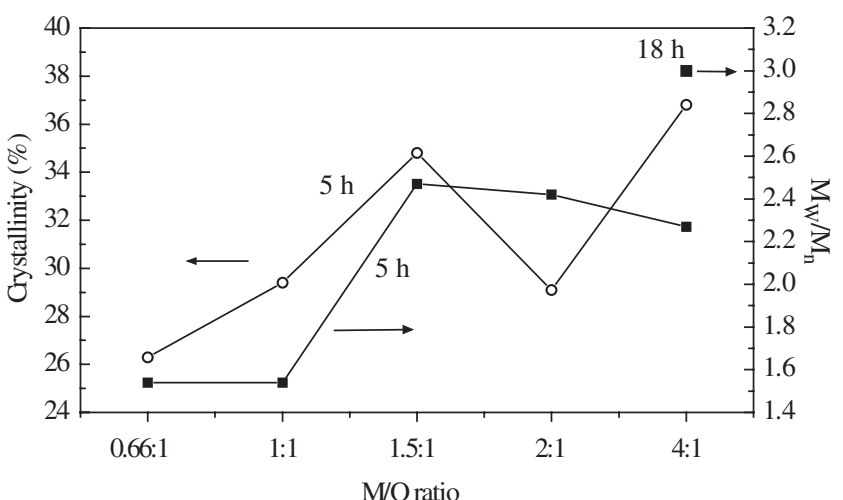

(b)

Figure 5. Values of the percentage of crystallinity of powdery PTOL doped with a $\mathrm{HCl} 1.0 \mathrm{~mol} \mathrm{~L}^{-1}$ solution as a function of the, a) molecular weight in THF; b) polydispersity $\left(\mathrm{M}_{\mathrm{w}} / \mathrm{M}_{\mathrm{n}}\right)$. 
consumption of the oxidant that was added to the reaction medium in an amount greater than that required by the reaction stoichiometry. After all these steps, the polymer formed in its completely oxidized state (pernigraniline) also contributes to the process of oxidation of monomers and oligomers, since it reduces itself to the emeraldine state at point $\mathbf{D}$ (final stage). This mechanism makes possible the growth of the polymer chain, which precipitates when the molecule becomes so large that it cannot remain in solution anymore. The results of MW as a function of the $\mathrm{M}: \mathrm{O}$ ratio can be explained assuming that in the case of a low relative concentration of the oxidant, the oxidant oxidizes the corresponding fraction of the monomer at the starting stage of the reaction, depleting it. If the concentration of radicals is low and the concentration of monomers high, there is a great probability of radicals meeting monomers and the molecular chain grows almost freely. This would explain the higher values of MW obtained at higher $\mathrm{M}: \mathrm{O}$ ratios. On the other hand, for a relatively high concentration of oxidant, the initial concentration of radical cations is large, and as a consequence, there is a great probability that radicals meet radicals, generating oligomers with a low MW. These neutral oligomers can be reoxidized by the remaining oxidant, but at the end, the final MW of the polymer obtained is still low.

It was also noticed that the reaction yield for PTOL increases with the relative amount of the oxidant added to the reaction medium, even if the relative amount of oxidant was in excess. By following the progress of the polymerization reaction, it can be realized that the amount of the monomer in the reaction medium at any time is always higher than that expected taking into account the amount of oxidant already consumed up to that time. These data indicate that the oxidant is consumed not only to oxidize the monomer, but also, species that are formed in the reaction medium. Such behavior can be explained assuming that the initially formed radical cations combine with themselves or monomers to form neutral species or radical oligomers, respectively. The neutral species are then re-oxidized by the oxidant demanding an excess of oxidant in order to oxidize more monomers and new neutral species. From the OCP curves, it was observed that the time at the $\mathrm{BC}$ plateau increases as the $\mathrm{M}: \mathrm{O}$ ratio decreases. As there is an excess of the oxidant in the reaction medium relative to the monomer, as the $\mathrm{M}: \mathrm{O}$ ratio decreases, it can be concluded that other species distinct from the monomer are being oxidized and contribute to the consumption of the oxidant. This is similar to the case of reaction yield as a function of the $\mathrm{M}: \mathrm{O}$ ratio, as discussed before.

\section{Conclusions}

The chemical synthesis of PTOL at five different M:O ratios was carried out in an aqueous $\mathrm{HCl}$ solution and results of $\mathrm{OCP}$, reaction yield, molecular weight (MW) and crystallinity were obtained. The IR results indicated that the chemical structure of PTOL is the same, independently of the M:O ratio used. On the other hand, despite the lower reaction yields obtained in such cases, PTOLs synthesized at higher M:O ratios displayed higher MW values and crystallinity. As a general feature, it can be asserted that the crystallinity of PTOL increases with increasing MW. However, after a prolonged exposure to the acid (dopant), the percentage of crystallinity is lower. Taking all these results into account, it is recommended that the synthesis of PTOL be performed with lower amounts of oxidant, although lower values of reaction yields are usually obtained under such synthesis conditions.

\section{References}

1. MacDiarmid AG. Sintetic metals: A novel role for organic polymers (Nobel lecture). Angewandte Chemie-International Edition. 2001; 40(14):2581-90.
2. Kvarnström C, Ivaska A. Handbook of Organic Conductive Molecules. Nalwa HS ed; 1997.

3. Mattoso LHC. Polianilinas: síntese, estrutura e propriedades. Química Nova. 1996; 19(4):388-99.

4. Gonçalves D, dos Santos DS, Mattoso LHC, Karasz FE, Akcelrud L, Faria RM. Poly(o-methoxyaniline): solubility, deprotonation-protonation process in solution and cast films. Synth Metals. 1997; 90(1):5-11.

5. Mattoso LHC, Faria RM, Bulhões LOS, MacDiarmid AG. Synthesis, doping and processing of high-molecular-weight poly(o-methoxyaniline). J Polymer Science Part A - Polymer Chem. 1994; 32(11):2147-53.

6. Cattarin S, Doubova L, Mengoli G, Zotti G. Eletrosynthesis and properties of ring-substituted polyanilines. Electrochimica Acta.1988; 38(8):1077-84.

7. Wei Y, Sun Y, Tang J. Auto-aceleration and kinetics of electrochemical polymerization of aniline. J Phys Chem. 1989; 93(12):4878-81.

8. Mohilner DM, Argersinger WJ, Adams RN. Investigation of kinetics and mechanism of anodic oxidation of aniline in aqueous sulfuric acid solution at a platinum electrode. J Am Chem Soc.1962; 84(19):3618, 1962.

9. Stejskal J, Kratochvíl P, Jenkins AD. The formation of polyaniline and the nature of its structures. Polymer. 1996; 37(2):367-9.

10. Tzou K, Gregory RV. Kinetic-study of the chemical polymerization of aniline in aqueous-solutions. Synth Metals. 1992; 47(3):267-77.

11. Wei Y, Tang X, Sun Y, Focke WW. A study of the mechanism of aniline polymerization. J Polymer Sci. 1989; 27(7):2385-96.

12. Shim YB, Park SM. Electrochemistry of conductive polymers.7.Autocatalytic rate-constant for polyaniline growth. Synth Metals. 1989; 29(1):E169-74.

13. Rodrigues MA, De Paoli MA. Electrochemical properties of chemically prepared poly(aniline). Synth Metals. 1991; 43(1-2):2957-62.

14. MacDiarmid AG, Chiang JC, Halpern M, Huang WS, Mu SL, Somasiri $\mathrm{NLD}, \mathrm{Wu} \mathrm{W}$, Yaniger SI. Polyaniline - interconversion of metallic and insulating forms. Mol Cryst Liq Cryst. 1985; 121(1-4):173-80.

15. MacDiarmid AG, Epstein AJ. Polyanilines: a novel class of conducting polymers. Faraday Discuss. 1989; 88:317.

16. Cao Y, Andreatta A, Heeger AJ, Smith P. Influence of chemical polymerization conditions on the properties of polyaniline. Polymer. 1989; 30(12):2305-11.

17. Adams PN, Laughlin PJ, Monkman AP. Synthesis of high molecular weight polyaniline at low temperatures. Synth Metals. 1996; 76(1-3):157-60.

18. Armes SP, Miller JF. Optimum reaction conditions for the polymerization of aniline in aqueous-solution by ammonium persulfate. Synth Metals. 1988; 22(4):385-93.

19. MacDiarmid AG, Manohar SK, Masters JG, Sun Y, Weiss H, Epstein AJ. Polyaniline - Syntesis and properties of pernigraniline base. Synth Metals. 1991; 41(1-2):621-26.

20. Wei Y, Hsueh KF, Jang GW. Monitoring the chemical polymerization of aniline by open-circuit-potential measurements. Polymer. 1994; 35(16):3572-5.

21. Mattoso LHC, Manohar SK, MacDiarmid AG, Epstein AJ. Studies on the chemical syntheses and the characteristics of polyaniline derivatives. J Polym Sci. 1995; 33(8):1227-34.

22. Fong Y, Schlenoff JB. Polymerization of aniline using mixed oxidizers. Polymer. 1995; 36(3):639-43.

23. Geng YH, Li J, Sun ZC, Jing XB, Wang FS. Polymerization of aniline in an aqueous system containing organic solvents. Synth Metals. 1998; 96(1):1-6.

24. Gomes MAB, Gonçalves D, De Sousa ECP, Valla B, Aegerter MA, Bulhões LOS. Solid-state electrochromic display based on polymer electrode polymer electrolyte interface. Electrochim Acta. 1992; 37(9):1653-6.

25. Wang YZ, Joo J, Hsu CH, Epstein EJ. Charge-transport of camphor sulfonic acid-doped polyaniline and poly(o-toluidine) fibers - role of processing. Synth Metals. 1995: 68(3):207-11.

26. Anand J, Palaniappan S, Sathyanarayana DN. Poly(o-and m-toluidine)polystyrene blends: spectral, termal and electrical properties. Polymer. 1998; 39(26):6819-25.

27. Manohar SK, MacDiarmid AG, Epstein AJ. Polyaniline - Pernigraniline, 
an isolable intermediate in the conventional chemical synthesis of emeraldine. Synth Metals 1991; 41(1-2):711-4.

28. Sun Y, MacDiarmid AG, Epstein AJ. Polyaniline - synthesis and characterization of pernigraniline base. J Chem Soc Chem Commun. 1990; (7):529-31.

29. Gazotti WA, De Paoli MA. High yield preparation of a soluble polyaniline derivative. Synth Metals. 1996; 80(3):263-9.

30. Oh EJ, Min Y, Wiesinger JM, Manohar SK, Scherr EM, Prest PJ, MacDiarmid AJ. Epstein AJ. Polyaniline - dependency of selected properties on molecular-weight. Synth Metals. 1993; 55(2-3):977-82.

31. Tang W, Sun Y, Wei Y. Molecular - weight of chemically polymerized polyaniline. Makromol Chem Rapid Commun. 1988; 9(12):829-34.

32. Wei Y, Hsueh KF, Tang X, Sun Y. Molecular weights of polyaniline and its derivatives. Polym Prepr(Am Chem Soc, Div Polym Chem). 1989; 30(1):226-7.

33. Liao YH, Kwei TK, Levon K. Investigation of the aggregation phenom- enon of polyaniline in dilute-solutions. Macromol. Chem Phys. 1995; 196(10):3107-16.

34. Yang D, Adams PN, Goering R, Mattes BR. New methods for determining the molecular weight of polyaniline by size exclusion chromatography. Synth Metals. 2003; 135(1-3):293-4.

35. Monkman AP, Adams P. Structural characterization of polyaniline free standins films. Synth Metals. 1991; 41(3):891-6.

36. Tang JS, Jing XB, Wang BC, Wang F. Infrared-spectra of soluble polyaniline. Synth Metals. 1988; 24(3):231-8.

37. Wang FS, Tang JS, Wang LX, Zhang HF, Mo ZS. Study on the crystallinity of polyaniline. Mol Cryst Liq Cryst. 1988; 160:175-84.

38. Chaudhari HK, Kelkar DS. X-Ray diffraction study of doped polyaniline. J Appl Polymer Sci. 1996; 62:15-18.

39. Manjunath BR, Venkatar A, Stephen T. Effect of moisture present in polymers on their x-Ray diffraction patterns. J Appl Polymer Sci. 1973; 17(4):1091-9. 